Bruna Ferreira Melo ${ }^{\mathrm{a}}$

(iD) https://orcid.org/0000-0001-7027-5796

Kionna Oliveira Bernardes Santos ${ }^{\mathrm{a}}$ (iD) https://orcid.org/0000-0003-3181-2696

Rita de Cássia Pereira Fernandes ${ }^{b}$ (D) https://orcid.org/0000-0002-3353-5365

a Universidade Federal da Bahia, Programa de Pós-graduação em Saúde, Ambiente e Trabalho. Salvador, BA, Brasil.

b Universidade Federal da Bahia, Departamento de Medicina Preventiva e Social, Programa de Pós-graduação em Saúde, Ambiente e Trabalho Salvador, BA, Brasil.

Contato:

Rita de Cássia Pereira Fernandes E-mail:

ritafernandes@ufba.br

Os autores declaram que o estudo está associado a uma bolsa proveniente da Coordenação de Aperfeiçoamento de Pessoal de Nível Superior (CAPES), e que não há conflitos de interesses.

Os autores informam que o trabalho não foi apresentado em evento científico.

Trabalho baseado na dissertação de mestrado de Bruna Ferreira Melo, intitulada “Absenteísmo por transtornos mentais em trabalhadores do setor judiciário", apresentada em 2019 ao Programa de Pós-graduação em Saúde, Ambiente e Trabalho, da Faculdade de Medicina da Bahia, da Universidade Federal da Bahia.
Recebido: 20/09/2019

Revisado: 19/05/2020

Aprovado: 19/06/2020

\section{Indicadores de absenteísmo por doença mental no setor judiciário: abordagem descritiva de uma coorte retrospectiva}

\author{
Absenteeism indicators due to mental disorders in the \\ judiciary branch: descriptive approach of a retrospective cohort
}

\section{Resumo}

Objetivo: descrever os indicadores de afastamento por transtornos mentais de servidores públicos do setor judiciário da Bahia. Métodos: estudo descritivo com dados de uma coorte retrospectiva sobre a casuística de absenteísmo-doença no período de 2011 a 2016. Foi traçado o perfil sociodemográfico e ocupacional, assim como foram estimados os indicadores epidemiológicos. $\mathrm{O}$ evento foi caracterizado a partir da frequência e duração dos afastamentos e dos diagnósticos clínicos. Resultados: registraram-se 1.023 eventos, com maior quantidade de episódios entre servidores com mais de 10 anos de serviço. As mulheres constituíram o grupo mais afetado, com prevalências superiores a dos homens em todo o período (76,50/1.000 trabalhadores em 2016). Em 2016, a incidência cumulativa foi de 12,72 casos novos/1.000 trabalhadores entre as mulheres e 5,58 casos novos/1.000 trabalhadores entre os homens. Os transtornos do humor, neuróticos e relacionados ao estresse foram os diagnósticos clínicos mais prevalentes associados ao absenteísmo-doença. Conclusão: o perfil e a dinâmica das ocorrências de absenteísmo-doença entre servidores públicos do setor judiciário da Bahia mostram semelhanças com outros serviços, validando sua externalidade e suscitando desafios para os serviços de saúde, principalmente quanto à detecção precoce do risco de afastamento e da monitorização das condições de trabalho.

Palavras-chave: absenteísmo; transtornos mentais; epidemiologia descritiva; saúde do trabalhador.

\begin{abstract}
Objective: to describe the indicators of sick leave due to mental disorders among civil servants of the Judiciary Branch in Bahia, Brazil. Methods: descriptive study conducted with data from a retrospective cohort on the casuistry of illnessrelated absenteeism from 2011 to 2016. We collected data on the participants' sociodemographic and occupational profile, and estimated their epidemiological indicators. The event was characterized based on the frequency and duration of absences, as well as the clinical diagnoses. Results: 1,023 absence events were recorded, most of which among civil servants in service for more than 10 years. Women were the most affected group, with higher prevalence than men in the entire period (76.50/1000 workers in 2016). In 2016, the cumulative incidence was 12.72 new cases/1000 workers among women and 5.58 new cases/1000 workers among men. Mood, neurotic, and stress-related disorders were the most prevalent clinical diagnoses associated with absenteeism. Conclusion: the profile and dynamics of the illness-related absenteeism among civil servants of the Judiciary Branch in Bahia are similar to those verified in other services, validating the externality of such events and posing challenges for health services, especially regarding early detection of sick leave risk, as well as working conditions monitoring.
\end{abstract}

Keywords: absenteeism; mental disorders; epidemiology, descriptive; occupational health. 


\section{Introdução}

O absenteísmo-doença configura-se no afastamento do indivíduo do trabalho por motivos de saúde, podendo indicar problemas existentes relacionados ao contexto de trabalho, além de desencadear consequências e danos não só para o trabalhador, mas também para a organização e a sociedade ${ }^{1,2}$.

Inserido em um cenário com diferentes dimensões interrelacionadas, que envolvem desde aspectos socioeconômicos até o próprio ambiente de trabalho, o absenteísmo-doença tem uma origem complexa e não pode ser explicado exclusivamente por problemas de saúde. Ainda assim, representa um marcador importante para o aumento da recidiva de ausência no trabalho, mortalidade e aposentadoria precoce ${ }^{1,3}$.

Um contexto de trabalho caracterizado por aumento da demanda e presença de estresse expõe o trabalhador a sobrecarga psicológica, tensão e alertas permanentes, que podem levá-lo a vivenciar experiências adoecedoras e o desenvolvimento de distúrbios psicofisiológicos relacionados ao ambiente ocupacional $^{4,5}$.

No Brasil, os transtornos mentais (TM) estão entre as principais causas de absenteísmo-doença e respondem pela duração mais longa das licenças, assim como representam um indicador de risco à incapacidade para o trabalho ${ }^{5-7}$. Além disso, apontam para uma interface do trabalho pouco explorada, que tem no estresse, assédio moral, conflitos com liderança, entre outros fatores, questões de ordem psicológica que podem prever eventos de afastamentos ${ }^{2,8}$.

As transformações tecnológicas, econômicas e culturais ocorridas no contexto operacional do trabalho têm pautado mudanças significativas na divisão do trabalho nas organizações, que podem influenciar condições ambientais relacionadas ao adoecimento mental ${ }^{9}$. Entre os trabalhadores do setor judiciário, por exemplo, o aumento da carga de trabalho e o esforço mental nos últimos anos oriundo das novas exigências impostas pelo estabelecimento de metas, inovações tecnológicas e modernização das atividades, têm tornado o ambiente do serviço público propício para o desenvolvimento de conflitos ${ }^{10-12}$.

Apesar da gravidade relativa à incapacidade atribuída a esses transtornos, a invisibilidade acerca do impacto deste agravo aponta para a necessidade de investigar eventos como o absenteísmo-doença ${ }^{13}$. A importância de investigações relacionadas a doenças crônicas como os TM se sustenta, sobretudo, pelo custo social envolvido, decorrente da exclusão dos indivíduos do trabalho ${ }^{14}$. Nesse sentido, a análise do absenteísmo-doença por TM contribui para o dimensionamento do problema e para a busca de fatores relacionados ao trabalho que sejam potencialmente estressantes, além de favorecer a identificação de possíveis cenários de exposição para o adoecimento por TM, aos quais os trabalhadores podem estar submetidos ${ }^{2,15}$.

Além disso, investigações a respeito desta temática ainda são incipientes e heterogêneas quanto aos indicadores utilizados para dimensionar esse evento em diversos países, devido a diferenças nas formas de mensuração e classificação do afastamento, o que reforça a necessidade de estudos sobre absenteísmo-doença por $\mathrm{TM}^{16}$.

Assim, este estudo teve como objetivo descrever os indicadores dos afastamentos por TM de servidores públicos do setor judiciário da Bahia.

\section{Métodos}

Trata-se de estudo descritivo com dados de uma coorte retrospectiva sobre a casuística de absenteísmo-doença por TM de trabalhadores do serviço judiciário, a partir de registros ocupacionais armazenados no Setor de Tecnologia da Informação e Comunicação (Setic) de um serviço do setor judiciário em Salvador, Bahia. As atividades desempenhadas no serviço em questão são do tipo judiciária e administrativa, em varas de trabalho, gabinetes, secretarias, seções, postos de segurança e núcleos de apoio, que se distribuem em municípios da região metropolitana de Salvador (4) e do interior do estado da Bahia (28 municípios).

O período da pesquisa compreendeu de 1 de janeiro de 2011 até 31 de dezembro de 2016, contemplando um censo de servidores públicos federais efetivos no cargo e ativos no banco de dados do estudo. Por ser uma coorte fixa, não foram admitidos novos indivíduos após o início do seguimento.

A atestação clínica da presença dos agravos foi realizada em sua maioria por profissionais externos à instituição, sendo homologadas pelo serviço interno, podendo também terem sido identificadas pelo serviço médico da organização. Excluíram-se os trabalhadores que estavam afastados por TM no banco de dados do estudo.

Definiram-se variáveis de interesse divididas em três tipos. O primeiro diz respeito às características sociodemográficas: sexo (masculino e feminino); idade (até 30 anos, $>30$ anos - ponto de corte adotado para melhor descrição da frequência da variável); e escolaridade (ensino fundamental completo, ensino médio completo, ensino superior completo). O segundo, características ocupacionais: área (administrativa, 
judiciária); cargo (analista, magistrado, técnico); e tempo de serviço (até 10 anos, > 10 anos). Por fim, características epidemiológicas: afastamentos identificados pelos códigos do grupo F, segundo a Classificação Internacional de Doenças e Problemas Relacionados à Saúde, $10^{\mathrm{a}}$ revisão (CID-10); quantidade de afastamentos (1 a 4, 5 a 8, 9 ou mais); e tempo total de afastamento (1 a 15, 16 a 30,31 a 60, 61 a 90, 91 dias ou mais).

Para a distribuição percentual dos diagnósticos de TM, foram consideradas as categorias: transtornos mentais devido ao uso de substâncias psicoativas (F10-F19), transtornos do humor (F30-F39), transtornos neuróticos e relacionados com estresse (F40-F48), e outros. A última categoria agrupou os seguintes diagnósticos: transtornos mentais orgânicos, inclusive os sintomáticos (F00-F09); esquizofrenia, transtornos esquizotípicos e transtornos delirantes (F20-F29); síndromes comportamentais associadas a disfunções fisiológicas e a fatores físicos (F50-F59); transtornos da personalidade e do comportamento do adulto (F60-F69); transtornos hipercinéticos (F90); e problemas relacionados com a organização de seu modo de vida (Z73).

O cálculo das prevalências anuais foi feito dividindo-se o total de casos de absenteísmo-doença por TM pela população exposta em cada ano. Incidências cumulativas (IC) foram calculadas dividindo-se o total de casos incidentes de afastamento por TM em cada ano pela população exposta no início do período e constante ao longo do tempo.

As etapas de tabulação e produção dos dados foram executadas com o auxílio dos programas Statistical Package for the Social Science (SPSS 21) e Microsoft Excel 2013.

O estudo foi aprovado pelo Comitê de Ética em Pesquisa da Faculdade de Medicina da Bahia sob protocolo $\mathrm{n}^{\circ}$ CAAE 6309.2916.3.0000.5577, em 19 de dezembro de 2016, e procedido nas normas da Resolução no 466/2012 do Conselho Nacional de Saúde.

\section{Resultados}

No período do estudo, foram observados 1.023 eventos de absenteísmo-doença decorrentes de TM. Para todas as variáveis, as maiores proporções ocorreram entre os trabalhadores que não se afastaram. No entanto, as proporções de afastamento observadas foram de 12,4\% entre o sexo feminino, 10,7\% entre aqueles com mais de 30 anos de idade, 10,2\% na área judiciária, 17,2\% entre os magistrados e $11,6 \%$ entre os trabalhadores com tempo de serviço acima de 10 anos (Tabela $\mathbf{1}$ ).

Tabela 1 Frequência das variáveis sociodemográficas e ocupacionais estudadas entre trabalhadores sem e com afastamento por transtornos mentais (TM) no setor judiciário da Bahia, 2011 a 2016

\begin{tabular}{|c|c|c|c|c|c|}
\hline \multirow{2}{*}{ Variável } & \multicolumn{2}{|c|}{ Sem afastamento } & \multicolumn{2}{|c|}{ Com afastamento } & \multirow{2}{*}{$\begin{array}{c}\text { Total } \\
n\end{array}$} \\
\hline & $n$ & $(\%)$ & $n$ & $(\%)$ & \\
\hline \multicolumn{6}{|l|}{ Sexo } \\
\hline Masculino & 1.163 & 93,0 & 88 & 7,0 & 1.251 \\
\hline Feminino & 1.235 & 87,6 & 174 & 12,4 & 1.409 \\
\hline \multicolumn{6}{|l|}{ Idade } \\
\hline$\leq 30$ anos & 410 & 94,5 & 24 & 5,5 & 434 \\
\hline$>30$ anos & 1.988 & 89,3 & 238 & 10,7 & 2.226 \\
\hline \multicolumn{6}{|l|}{ Escolaridade } \\
\hline Ensino fundamental completo & 43 & 89,6 & 5 & 10,4 & 48 \\
\hline Ensino médio completo & 361 & 89,6 & 42 & 10,4 & 403 \\
\hline Ensino superior completo & 1.994 & 90,3 & 215 & 9,7 & 2.209 \\
\hline \multicolumn{6}{|l|}{ Área } \\
\hline Administrativa & 1.010 & 90,6 & 104 & 9,4 & 1.114 \\
\hline Judiciária & 1.388 & 89,8 & 158 & 10,2 & 1.546 \\
\hline \multicolumn{6}{|l|}{ Cargo } \\
\hline Analista & 746 & 91,4 & 70 & 8,6 & 816 \\
\hline Magistrado & 180 & 82,2 & 39 & 17,8 & 219 \\
\hline Técnico & 1.472 & 90,6 & 153 & 9,4 & 1.625 \\
\hline \multicolumn{6}{|l|}{ Tempo de serviço } \\
\hline$\leq 10$ anos & 846 & 93,5 & 59 & 6,5 & 905 \\
\hline$>10$ anos & 1.552 & 88,4 & 203 & 11,6 & 1.755 \\
\hline
\end{tabular}


Entre os afastamentos, vale destacar que a maioria das licenças por TM foi observada em trabalhadores da área judiciária, em vez da administrativa. Não houve variação expressiva de afastamentos na categoria escolaridade, entretanto os maiores percentuais encontram-se entre os trabalhadores com ensino fundamental completo e ensino médio completo: 10,5\% (Tabela 1).

Com relação à frequência de afastamentos (Tabela 2), observa-se que a maioria dos trabalhadores apresentaram de 1 a 4 licenças durante o período analisado $(76,7 \%)$. Para o tempo total de afastamento (Tabela 2), que corresponde à soma dos dias em absenteísmo-doença de todas as licenças por TM por indivíduo, observa-se que a maior parte dos trabalhadores apresentaram de 1 a 15 dias de afastamento por absenteísmo-doença $(48,1 \%)$, seguidos por aqueles que totalizaram mais de 90 dias em todo o período analisado (23,7\%).

A partir da demanda de afastamentos analisada e considerando a possibilidade da existência de casos do evento por doença mental anteriores ao início da coorte, durante o período analisado houve redução da prevalência de trabalhadores afastados, com destaque para o sexo masculino, que apresentou uma variação de 51,83 casos/1.000 trabalhadores em 2011 para 31,97 casos/1.000 trabalhadores em 2016. Contudo, em todo o período, foram verificados indicadores epidemiológicos elevados para o absenteísmo-doença por TM. Em todos os anos, as mulheres constituíram o grupo mais afetado com quantidade de afastamentos superiores às dos homens, variando de 114,16 casos/1.000 trabalhadores para 76,50 casos/1.000 trabalhadores, entre 2012 e 2016, respectivamente (Figura 1).

Além disso, a análise da IC, com base na observação dos casos de absenteísmo-doença por TM registrados a partir de 2011 apresentou, nesse ano, o indicador de 41,87 casos novos/1.000 trabalhadores do sexo feminino e 24,72 casos novos/a cada 1.000 trabalhadores do sexo masculino. Em 2016, foram registrados 12,72 casos novos/1.000 trabalhadores entre as mulheres e 5,58 casos novos por mil trabalhadores entre os homens (Figura 2).

Tabela 2 Frequência e duração de afastamento dos trabalhadores em absenteísmo-doença por transtornos mentais (TM) no setor judiciário da Bahia, 2011 a 2016

\begin{tabular}{lcc}
\hline \multicolumn{1}{c}{ Variável } & $(n=262)$ & $(\%)$ \\
\hline Frequência do afastamento por TM & 201 & 76,7 \\
1 a 4 afastamentos & 30 & 11,5 \\
5 a 8 afastamentos & 31 & 11,8 \\
9 afastamentos ou mais & & \\
Mediana: 2 & & \\
Valor máximo: 35 & & \\
Valor mínimo: 1 & 126 & 48,1 \\
Duração total do afastamento por TM* & 33 & 12,6 \\
1 a 15 dias & 25 & 9,5 \\
16 a 30 dias & 16 & 6,1 \\
31 a 60 dias & 62 & 23,7 \\
61 a 90 dias & & \\
90 dias ou mais & & \\
Mediana: 20,5 & & \\
Valor máximo: 975 & & \\
Valor mínimo: 1 & & \\
\hline soma dos dias em absenteísmo-doença de todas as licenças por TM, por indivíduo. &
\end{tabular}




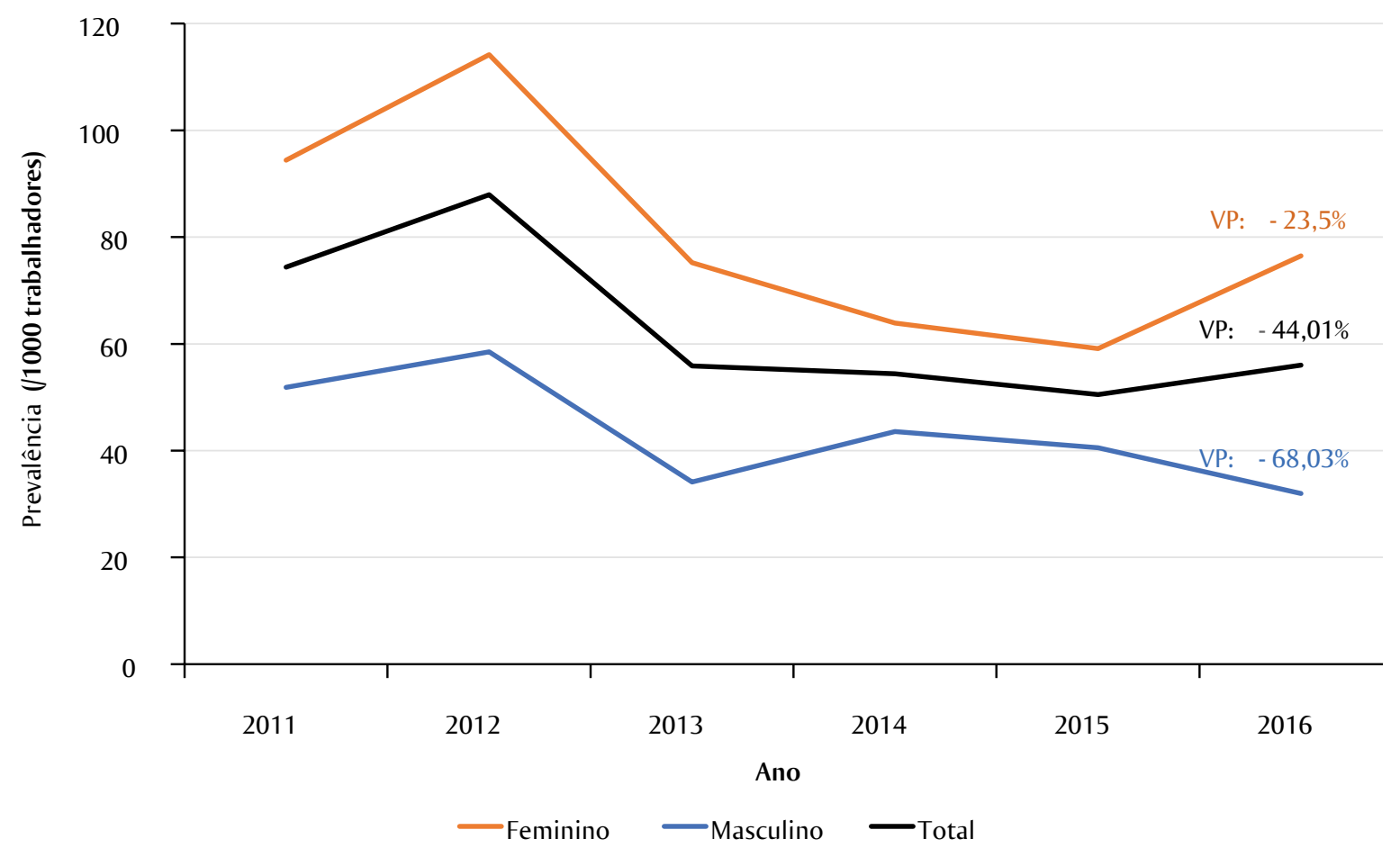

Figura 1 Prevalência anual de absenteísmo-doença por transtornos mentais (TM) entre trabalhadores do setor do judiciário da Bahia, 2011 a 2016

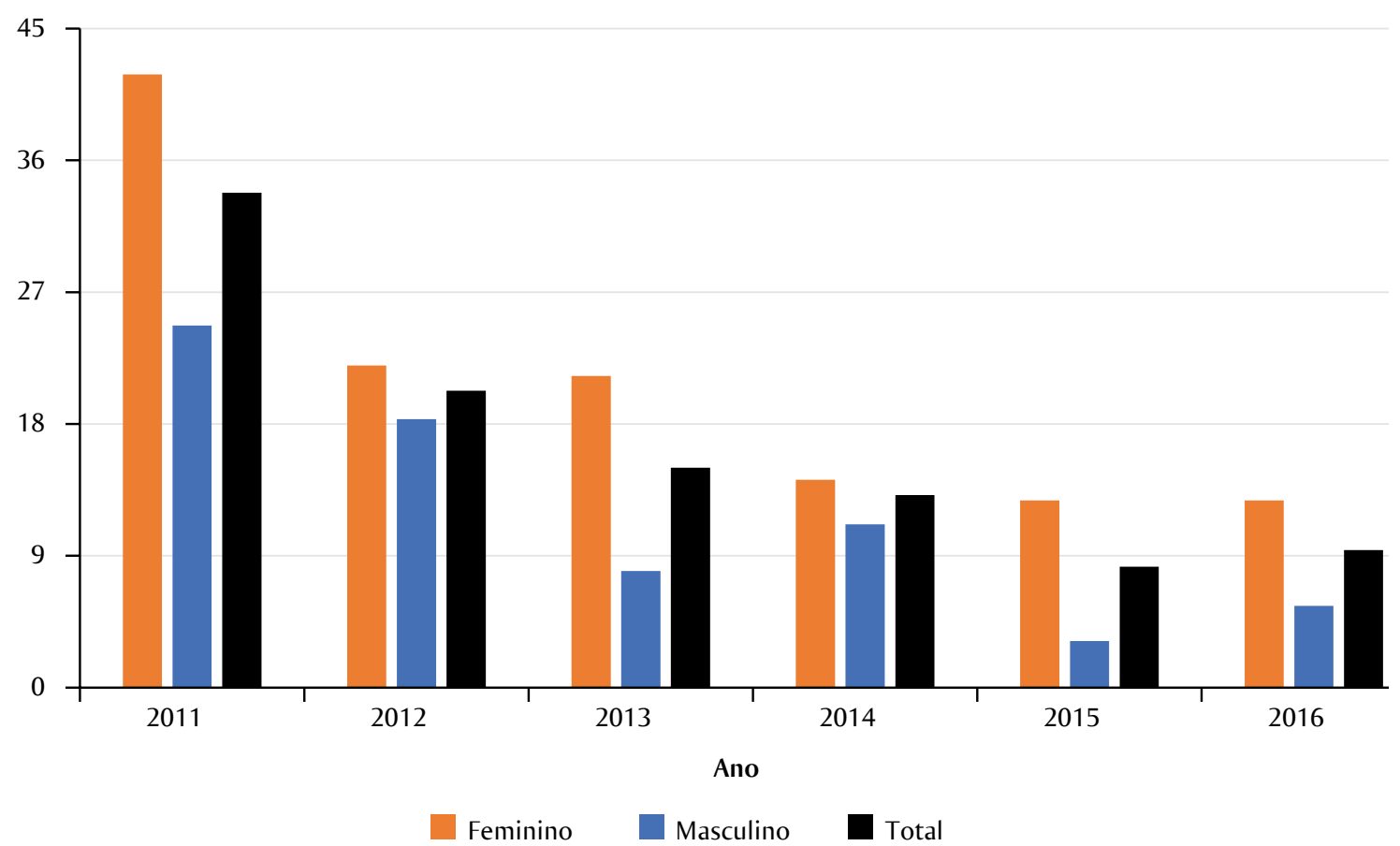

Figura 2 Incidência cumulativa anual de absenteísmo-doença por transtornos mentais (TM) entre trabalhadores do setor judiciário da Bahia, 2011 a 2016. 
A distribuição dos diagnósticos de TM referentes à totalidade de eventos ao longo do tempo revelou uma predominância dos transtornos do humor e dos transtornos neuróticos e relacionados com o estresse como as causas mais frequentes para o absenteísmo-doença. Por ano, cerca de 45,4\% do total dos afastamentos foi atribuído aos transtornos de humor, enquanto os transtornos neuróticos e relacionados com o estresse responderam por 46,5\%. Além desses, identificou-se também a presença de diagnósticos de TM devido ao uso de substância psicoativa ao longo do tempo, mesmo que discretamente, correspondendo a menos de $5 \%$ dos eventos em todos os anos, com tendência de crescimento. Outras categorias do grupo F (CID-10) também foram verificadas, porém em frequências menores (Figura 3).

Com relação à duração dos afastamentos, no entanto, constatou-se que os diagnósticos de esquizofrenia, transtornos esquizotípicos e transtornos delirantes (F20-F29), transtornos do comportamento e transtornos emocionais da infância ou adolescência (F90-F99) e as síndromes comportamentais associadas a disfunções fisiológicas e a fatores físicos (F50-F59) apresentaram as maiores médias de dias de afastamento, quando comparados às demais situações clínicas: 56,69 dias, 51,75 dias e 46,43 dias, respectivamente.

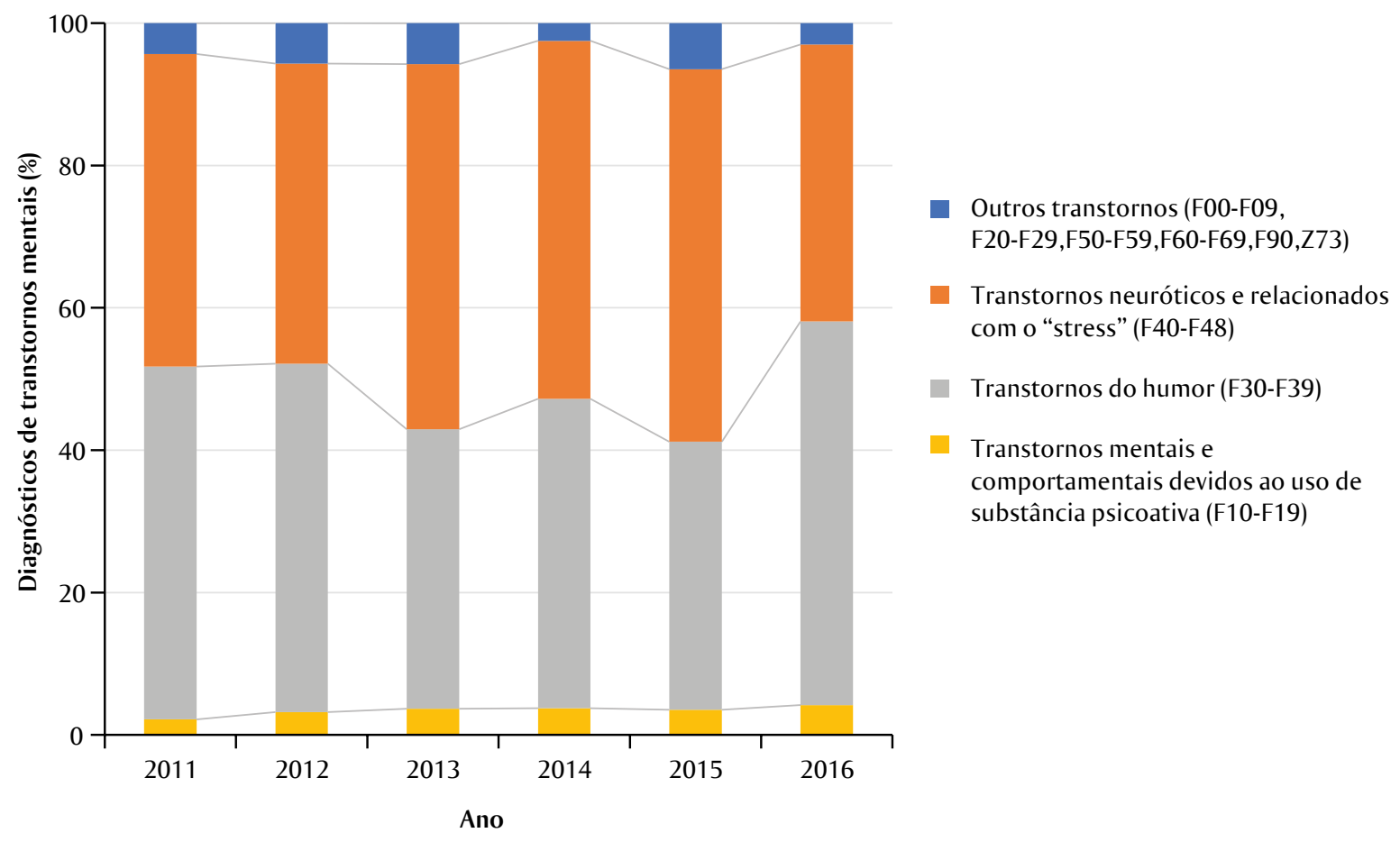

Figura 3 Distribuição anual dos principais diagnósticos clínicos - Grupo F/CID-10, para todos os casos de absenteísmo-doença entre servidores do judiciário da Bahia, 2011 a 2016

\section{Discussão}

Neste estudo foi possível estimar a magnitude do absenteísmo-doença por TM em servidores do judiciário durante o período de 2011 a 2016, observar a dinâmica da incidência do evento ao longo do tempo, além de verificar as entidades clínicas mais presentes nos diagnósticos. De forma geral, a população não apresentou alta frequência de absenteísmo-doença por doença mental nesse período, quando comparada com outros estudos sobre o mesmo evento $^{17-19}$. Ainda assim, as maiores proporções de afastamento ocorreram entre trabalhadores do sexo feminino, com idade acima de 30 anos, com ensino fundamental e médio completo, pertencentes a área judiciária, com cargo de magistrado e mais de 10 anos de serviço.

A maior prevalência de absenteísmo-doença por TM em mulheres encontrada neste estudo corrobora outros achados na literatura referentes a investigações sobre sofrimento psíquico ${ }^{2,5,6,20,21}$. A abrangência desses agravos entre as mulheres é evidenciada em países desenvolvidos e subdesenvolvidos, nos quais os transtornos depressivos estão entre as principais causas de adoecimento, e representam preditores de absenteísmo-doença quando existem fatores estressores na organização de trabalho ${ }^{5,22}$. 
A predisposição do sexo feminino a tensões psicológicas pode ser explicada do ponto de vista contextual. Alguns autores afirmam que, devido ao duplo papel de trabalho, dentro e fora de casa, que exercem na sociedade, as mulheres já se encontram em estado de cansaço mental ${ }^{23}$. Entretanto, ressalta-se que o absenteísmo-doença por TM é um evento de atestação médica, ou seja, a maior proporção de licenças de trabalhadores do sexo feminino reflete o fato de que o processo de reconhecimento da doença e da procura por serviços médicos é culturalmente mais característico das mulheres, ajudando a explicar os resultados encontrados ${ }^{24}$.

Apesar da complexidade causal do absenteísmo-doença, a alta prevalência anual de afastamento por TM na população em geral pode levar ao questionamento sobre o impacto das formas de organização dos processos de trabalho na saúde dos trabalhadores. Na tentativa de atender às demandas impostas, os trabalhadores já adoecidos são induzidos a permanecer em atividade até que ocorra a percepção da incapacidade temporária, situação que caracteriza o presenteísmo, referido por vários autores, e que pode estar refletido na menor magnitude do absenteísmo-doença por doença mental aqui encontrado ${ }^{25,26}$.

Do ponto de vista do cargo, o magistrado se destacou em relação às categorias de analista e técnico, apresentando a maior proporção de afastamentos por TM. Estudos que abordam a saúde do magistrado e a complexidade de sua ocupação ainda são escassos, no entanto alguns já identificaram a presença de estressores e diagnósticos de ansiedade e depressão entre trabalhadores desta categoria e apontam para a necessidade de estratégias de prevenção relacionadas à saúde mental ${ }^{27-29}$.

Os custos sociais do presenteísmo vinculados ao adoecimento mental o tornam um desafio a ser enfrentado, bem como um fator que aponta para a complexidade do absenteísmo-doença a partir do reconhecimento de questões adversas - incluindo despesas médicas e farmacêuticas por longos períodos e a perda da funcionalidade para o trabalho -, que circundam o contexto do absenteísmo-doença e podem desencadear a exclusão social dos indivíduos afetados ${ }^{30,31}$.

Identificou-se, neste estudo, uma maior proporção de trabalhadores acima dos 30 anos, inclusive entre os funcionários afastados. Apesar do ponto de corte adotado para faixa etária não discriminar estratos mais avançados, a maioria dos trabalhadores afastados se concentrou na faixa entre 40 e 50 anos. A maior presença de idades mais avançadas tem sido descrita como reflexo da evolução demográfica na maioria dos países e, consequentemente, das políticas governamentais, as quais permitem que o trabalhador passe a maior parte da vida de forma ativa, mesmo em um único vínculo, como é o caso do servidor público, sujeito deste estudo ${ }^{32}$. Observou-se no estudo uma tendência de aumento de afastamento de trabalhadores mais velhos em comparação com aqueles de até 30 anos, o que pode ser explicado pelo processo de incapacidade crônica, assim como a necessidade de maior tempo de recuperação antes de retornar ao trabalho, associado ao envelhecimento ${ }^{32}$.

Com relação à escolaridade, o absenteísmo-doença apresentou particularidades; entre os indivíduos com ensino fundamental e médio completos, a frequência de afastamentos foi de 10,4\%, enquanto entre trabalhadores com nível superior, foi de apenas 9,7\%. Desta forma, o resultado corrobora outros estudos que indicam uma relação inversa entre escolaridade e propensão ao absenteísmo-doença, queixas depressivas e baixo status psicossocial no trabalho ${ }^{25,32-34}$, ou seja, quanto maior o nível de instrução, menor a inclinação para o afastamento e para os problemas de ordem mental. No entanto, alguns desses estudos revelam a necessidade de esclarecer melhor a relação entre esta variável e o desfecho ${ }^{5,24,33}$. Vale destacar que quando se observam apenas os trabalhadores afastados, nota-se uma predominância de servidores que tinham nível universitário completo, o que reflete a conjuntura desta população.

O estudo apresentou lacunas quanto às informações para caracterização do contexto de trabalho, comuns em se tratando de fontes de dados secundárias. Ainda assim, é preciso considerar que os TM são doenças de padrão crônico, sugerindo que, embora o adoecimento por TM não esteja associado exclusivamente ao contexto, condições de trabalho desfavoráveis podem indicar fatores que influenciam a decisão de se afastar ${ }^{35}$.

A análise da influência das condições de trabalho no problema do absenteísmo-doença será possível somente a partir da consideração das características da organização do trabalho do setor público como porta de entrada para as demandas da população. Apesar das especificidades do setor público, como gozar de estabilidade, dinamicidade das atividades e amplo relacionamento interpessoal, é possível que fatores como falta de controle sobre as tarefas, excessiva burocratização das atividades, insuficiência de condições favoráveis ao desenvolvimento de um trabalho compatível com as exigências sociais, impossibilidade de ascensão profissional e perda de sentido do trabalho possam funcionar como estressores psicológicos, compondo modelos de organização do trabalho que deterioram o estado geral de saúde autopercebido e aumentam a fadiga mental dos trabalhadores, que passam a adoecer ${ }^{9,24}$. 
Observaram-se maiores frequências anuais de diagnósticos de TM do grupo F (CID-10), relativos aos transtornos do humor e neuróticos, relacionados com o estresse e somatoformes. A predominância desses diagnósticos também foi verificada em outros estudos sobre o mesmo evento em populações semelhantes ${ }^{36-38}$. Estas condições referem-se a transtornos desencadeados por elementos sintomatológicos de depressão, ansiedade e estresse, que apesar da difícil identificação, especialmente quando em estado comórbido, afetam o funcionamento emocional, cognitivo e social do indivíduo e, possivelmente, estão associados a episódio incidente de absenteísmo-doença ${ }^{39,40}$.

Por fim, este estudo se preocupou com a inclusão de dados de absenteísmos-doença oriundos de registros oficiais da instituição e não de informações autorrelatadas, além dos diagnósticos dos casos provirem de atestados clínicos, o que garante a presença dos agravos, ao invés de condições de estado momentâneo, reduzindo a possibilidade de viés de informação. Além disso, o uso da incidência cumulativa (IC) coloca o estudo em alinhamento com a literatura quanto ao uso desse indicador como medida de risco de absenteísmo-doença durante um período específico ${ }^{40}$. Como limitações, vale destacar o fato de a maior parte das licenças terem sido atestadas por serviços fora da instituição onde os funcionários trabalhavam, o que pode ter levado a uma subestimação dos indicadores que dimensionaram o evento, assim como a carência de informações sobre a organização do trabalho, dificuldade encontrada em estudos dependentes de informações administrativas, prejudicando uma possível avaliação do contexto ocupacional.

\section{Conclusão}

Esta investigação contribuiu com informações empíricas que caracterizaram a ocorrência do absenteísmo-doença por TM em trabalhadores do setor judiciário da Bahia no período de 2011 a 2016, além de traçar o perfil sociodemográfico e ocupacional da população afetada e não afetada pelo evento. O padrão de funcionamento do serviço público permitiu vislumbrar semelhanças com outros serviços, o que torna a abordagem e as considerações válidas e com externalidade. Por fim, o estudo identificou também desafios para os serviços de saúde das instituições, quanto a iniciativas a serem desenvolvidas para proteção da saúde dos trabalhadores, tendo em vista a detecção precoce do risco de afastamento, a monitorização das condições de trabalho e o enfrentamento dos problemas incapacitantes já instalados.

\section{Contribuições de autoria}

Melo BF, Santos KOB e Fernandes RCP contribuíram substancialmente na concepção do estudo, no levantamento, análise e interpretação dos dados, na elaboração e revisões críticas do manuscrito, na aprovação da versão final e assumem responsabilidade integral pelo trabalho realizado e conteúdo publicado.

\section{Referências}

1. Goorts K, Vandenbroeck S, Rusu D, Du Bois $\mathrm{M}$, Godderis L. Screening for the risk on long-term sickness absence. J Public Health Res. 2018;7(2):1419.

2. Yaacob SS, Shaarial SZM, Noor NM, Ismail KI, Selvaraju R, Gani HA. Prevalence of sickness absence and its sociodemographic and occupational factors in a public service organization. KnE Life Sciences. 2018;4(5):687-96.

3. Ferreira RC, Griep RH, Fonseca MJM, Rotenberg L. A multifactorial approach to sickness absenteeism among nursing staff. Rev Saude Publica. 2012;46(2):259-68.

4. Franco T, Druck G, Seligmann-Silva E. As novas relações de trabalho, o desgaste mental do trabalhador e os transtornos mentais no trabalho precarizado. Rev Bras Saude Ocup. 2010;35(122):229-48.
5. Baasch D, Trevisan RL, Cruz RM. Perfil epidemiológico dos servidores públicos catarinenses afastados do trabalho por transtornos mentais de 2010 a 2013. Cien Saude Colet. 2017;22(5):1641-50.

6. Sala A, Carro ARL, Correa AN, Seixas PHD. Licenças médicas entre trabalhadores da Secretaria de Estado da Saúde de São Paulo no ano de 2004. Cad Saude Publica. 2009;25(10):2168-78.

7. Silva-Junior JS, Fischer FM. Afastamento do trabalho por transtornos mentais e estressores psicossociais ocupacionais. Rev Bras Epidemiol. 2015;18(4):735-44.

8. Holmgren K, Fjallstrom-Lundgren M, Hensing G. Early identification of work-related stress predicted sickness absence in employed women with musculoskeletal or mental disorders: a prospective, longitudinal study in a primary health care setting. Disabil Rehabil. 2013;35(5):418-26. 
9. Ribeiro CVS, Mancebo D. O servidor público no mundo do trabalho do século XXI. Psicol Cienc Prof. 2013;33(1):192-207.

10. Fonseca RMC, Carlotto MS. Saúde mental e afastamento do trabalho em servidores do judiciário do estado do Rio Grande do Sul. Psicol Pesq. 2011;5(2):117-25.

11. Dal Pai D, Lautert L, Tavares JP, Souza Filho GA, Dornelles RAN, Merlo ARC. Repercussões da aceleração dos ritmos de trabalho na saúde dos servidores de um juizado especial. Saude Soc. 2014;23(3):942-52.

12. Antloga CS, Maia M, Cunha KR, Peixoto J. Contexto de trabalho e custo humano no trabalho em um órgão do poder judiciário brasileiro. Cien Saude Colet. 2014;19(12):4787-96.

13. Brasil. Ministério da Saúde. Secretaria de Atenção à Saúde. Departamento de Ações Programáticas Estratégicas. Saúde mental [Internet]. Brasília, DF: Ministério da Saúde; 2015 [citado em 12 nov 2018]. (Caderno HumanizaSUS; vol. 5). Disponível em: http://bvsms.saude.gov.br/bvs/publicacoes/ saude_mental_volume_5.pdf

14. Silva-Junior JS, Fischer FM. Adoecimento mental incapacitante: benefícios previdenciários no Brasil entre 2008-2011. Rev Saude Publica. 2014;48(1):186-90.

15. Traesel ES, Merlo ARC. "Somos sobreviventes": vivências de servidores públicos de uma instituição de seguridade social diante dos novos modos de gestão e a precarização do trabalho na reforma gerencial do serviço público. Cad Psicol Soc Trab. 2014;17(2):224-38.

16. Dewa CS, Loong D, Bonato S, Hees H. Incidence rates of sickness absence related to mental disorders: a systematic literature review. BMC Public Health. 2014;14:205.

17. Zechinatti AC, Belloti JC, Moraes VY, Albertoni WM. Occupational musculoskeletal and mental disorders as the most frequent associations to worker's sickness absence: a 10-year cohort study. BMC Res Notes. 2012;5:229.

18. Fernandes MA, Ribeiro HKP, Santos JDM, Monteiro CFS, Costa RS, Soares RFS. Prevalence of anxiety disorders as a cause of workers' absence. Rev Bras Enferm. 2018;71(Suppl 5):2213-20.

19. Baptista MC, Burton WN, Nahas AK, Wang YP, Viana MC, Andrade LH. Absenteeism and presenteeism associated with common health conditions in Brazilian workers. J Occup Environ Med. 2019;61(4):303-13.

20. Ishtiak-Ahmed K, Perski A, Mittendorfer-Rutz E. Predictors of suicidal behaviour in 36,304 individuals sickness absent due to stress-related mental disorders - a Swedish register linkage cohort study. BMC Public Health. 2013;13:492.

21. Jarman L, Martin A, Venn A, Otahal P, Taylor $\mathrm{R}$, Teale B, et al. Prevalence and correlates of psychological distress in a large and diverse public sector workforce: baseline results from Partnering Healthy@Work. BMC Public Health. 2014;14:125.

22. Knuth BS, Silva RA, Oses JP, Radtke VA, Cocco RA, Jansen K. Mental disorders among health workers in Brazil. Cien Saude Colet. 2015;20(8):2481-8.

23. Jeyapal O. Nature of absenteeism among women workers of textile mills in Theni District. Shanlax International Journal of Economics. 2018;6(4):10-6.

24. Silva-Junior JS, Fischer FM. Long-term sickness absence due to mental disorders is associated with individual features and psychosocial work conditions. PLoS One. 2014;9(12):e115885.

25. Demerouti E, Le Blanc PM, Bakker AB, Schaufeli WB, Hox J. Present but sick: a three-wave study on job demands, presenteeism and burnout. The Career Development International. 2009;14(1):50-68.

26. Pinheiro M, Ivandic I, Razzouk D. The economic impact of mental disorders and mental health problems in the workplace. In: Razzouk D, editor. Mental health economics. Cham: Springer; 2017. p. $415-30$.

27. Lipp MEN, Tanganelli MS. Stress e qualidade de vida em Magistrados da Justiça do Trabalho: diferenças entre homens e mulheres. Psicol Reflex Crit. 2002;15(3):537-48.

28. Perales A, Chue H, Padilla A, Barahona L. Estrés, ansiedad y depresión en magistrados de Lima, Perú. Rev Peru Med Exp Salud Publica. 2011;28(4):581-8.

29. Costi CG. A saúde do magistrado: um breve levantamento bibliográfico. Revista da Esmesc. 2013;20(26):203-22.

30. Bryngelson A. Long-term sickness absence and social exclusion. Scand J Public Health. 2009;37(8):839-45.

31. Nagata T, Mori K, Ohtani M, Nagata M, Kajiki $\mathrm{S}$, Fujino Y, et al. Total health-related costs due to absenteeism, presenteeism, and medical and pharmaceutical expenses in Japanese employers. J Occup Environ Med. 2018;60(5):e273-80.

32. Cornelius LR, van der Klink JJL, Groothoff JW, Brouwer S. Prognostic factors of long term disability due to mental disorders: a systematic review. J Occup Rehabil. 2011;21(2):259-74.

33. Rabacow FM, Levy RB, Menezes PR, Luiz OC, Malik AM, Burdorf A. The influence of lifestyle and gender on sickness absence in Brazilian workers. BMC Public Health. 2014;14:317.

34. Aminian O, Feghhi L, Sharifian A, Eftekhar S. The relation between work-related psychosocial factors and development of depression among administrative staff. Galore International Journal of Health Sciences and Research. 2017;2(2):11-9.

35. Fragalá GE. O ambiente de trabalho e o adoecimento do trabalhador. In: Razzouk D, Lima MGA, Cordeiro Q, organizadores. Saúde mental e trabalho. São Paulo: Conselho Regional de Medicina do Estado de São Paulo; 2015. p. 91-110. 
36. Cruz CRB, Shirassu MM, Barbosa PLQ, Santana AMR. Transtornos mentais como causa de absenteísmo entre servidores públicos em São Paulo. Rev Psiquiatr Clin. 2011;38(6):265-6.

37. Roelen CAM, Norder G, Koopmans PC, van Rhenen W, van der Klink JJL, Bultmann U. Employees sicklisted with mental disorders: who returns to work and when? J Occup Rehabil. 2012;22(3):409-17.

38. Leão ALM, Barbosa-Branco A, Rassi Neto E, Ribeiro CAN, Turchi MD. Sickness absence in a municipal public service of Goiânia, Brazil. Rev Bras Epidemiol. 2015;18(1):262-77.

39. Kasper S. Anxiety disorders: under-diagnosed and insufficiently treated. Int J Psychiatry Clin Pract. 2006;10(Suppl 1):3-9.

40. Knudsen AK, Harvey SB, Mykletun A, Øverland S. Common mental disorders and long-term sickness absence in a general working population. The Hordaland Health Study. Acta Psychiatr Scand. 2013;127(4): 287-97. 